\title{
KERNFORSCHUNGSANLAGE JÜLICH GmbH
}

\section{Anomalous Impurity Transport in Plasmas}

by

A. Rogister, G. Hasselberg

Jül - 1846

Mai 1983

ISSN 0366-0885 


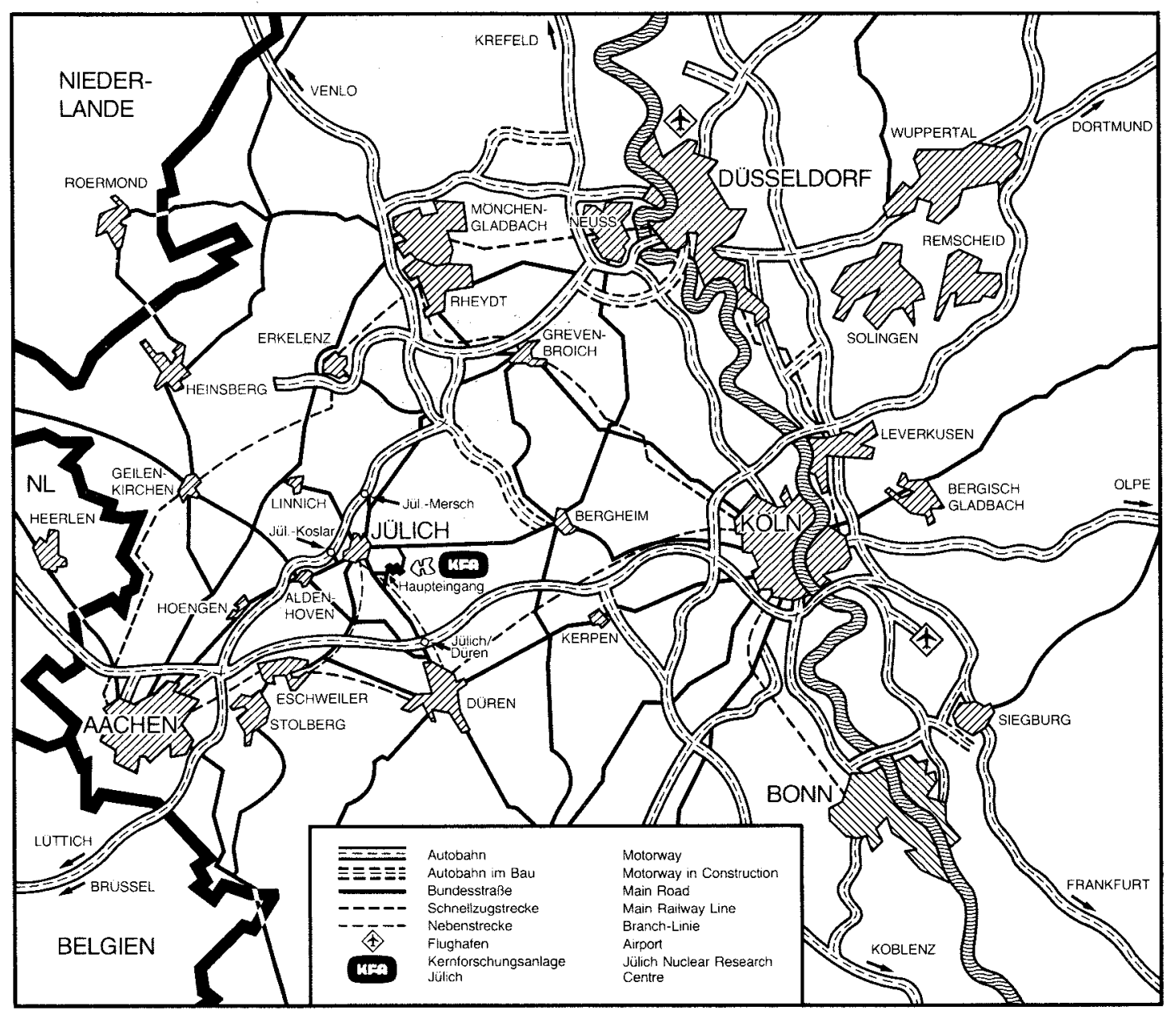

Als Manuskript gedruckt

Berichte der Kernforschungsanlage Jülich - Nr. 1846

Institut für Plasmaphysik Association EURATOM-KFA, Jül - 1846

Zu beziehen durch: ZENTRALBIBLIOTHEK der Kernforschungsanlage Jülich GmbH Postfach 1913 - D-5170 Jülich (Bundesrepublik Deutschland)

Telefon: 02461/610 Telex: 833556 kf d 


\section{Anomalous Impurity Transport in Plasmas}

by

A. Rogister, G. Hasselberg 

ANOMALOUS IMPURITY TRANSPORT IN PLASMAS

André Rogister and Günter Hasselberg

Institut für Plasmaphysik der Kernforschungsanlage Jülich GmbH

Association EURATOM-KFA, 5170 Jülich, Federal Republic of Germany

The transport of hith $\mathrm{Z}$ impurities resulting from Compton and induced scattering by drift waves is found to be comparable in magnitude with base ion and electron transport when the fluctuation spectrum is obtained from a recent theory of drift turbulence. The anomalous frictional flux is marginally subdominant whilst the diffusion flux is enhanced over the neoclassical value by a numerical factor which (i) agrees quantitatively with the results of impurity injection experiments, (ii) decreases with increasing base ion atomic mass, and (iii) is independent from the impurity charge to mass ratio. The theory further predicts a dramatic increase of the anomaly, a1so in agreement with experimental results, when the growth rate is enhanced within the heat pulse released by the sawtooth relaxations of the core. 
The present work continues our research effort on the transport properties of drift waves. These are considered to be the most probable candidates for causing anomalous transport in the gradient layer which surrounds the dense and hot core of magnetically confined plasmas and hopefully screens it from wall contamination. It is shown that the transport of impurities by the dissipative trapped electron instability is consistent with experiments when the spectra are derived from our recent works on drift turbulence /1-3/. The latter were developed on the premise that Compton and induced scattering, i.e. nonlinear ion Landau damping, are the dominant saturation processes.

The important and novel feature of that theory was to predict a nonlocal energy transfer from the long to the short ends of the one-dimensional poloidal spectrum. Nonlocal (distant) interactions are possible indeed as well as local (close) ones because of the bi-correspondence between the frequencies $\omega$ of drift waves and their poloidal mode numbers $k_{\theta}$. The process of nonlocal energy transfer explains well the position of the maximum of the observed spectra /4-7/; energy cascade, or local transfer, provides the correct spectral index at high mode numbers $/ 8 /$. The predicted short wavelength branch of the fluctuation spectrum which is maintained filled by the distant interactions yields the dominant contribution to the turbulent heat transport /9-10/. This permits to resolve a puzzling contradiction of Tokamak discharges: i.e. (1) the measured turbulence, which at present includes relatively long wavelengths only, is insufficient to account for all the anomalous losses calculated from the power balance equation $/ 11 /$, and (2) the profiles are always close to marginal stability with respect to the dissipative trapped electron modes implying that these are efficient transport agents /12/, once destabilized. As a logical consequence of this efficiency, which is verified by our 
theory, we have finally proposed in $/ 9,10 /$ that the quenching of the instability, e.g. at high densities, would lead to overheating of the core and to cooling of the periphery. Sawtooth relaxations of the core (periodic disruptions) and major disruptions would then occur once the released heat pulse is able to excite the instability along its path and thereby sustain its own transport in the same manner as a collisionless shock wave /13-15/. The high density limit obtained in this way agrees remarkably well with empirical scaling laws $/ 16 /$.

We demonstrate here that the diffusive flux of high $\mathrm{Z}$ impurities arises predominantly from nonlocal Compton and induced scattering of drift waves and is comparable, in moderately dense and dense plasmas to the base ion transport. The anomalous frictional flux plays a marginally subdominant role, though it is of the same size as the diffusive flux; it is contributed both by local and nonlocal scattering. (The frictional flux is proportional to the gradient of the base ion density and is usually directed towards the plasma core; the diffusive flux is proportional to the impurity density gradient.) These results agree well with the conclusions of the impurity injection experiments by Marmar et al. /17,18/ and by the TFR Group /19/. During the abrupt decay of the sawteeth according to our calculations - the anomalous impurity fluxes should increase even more dramatically than the heat fluxes which, however, must then sweep across the plasma all the energy accumulated in the core during the slow rise. This prediciton is consistent with the observed but not understood cleaning action of the sawteeth and, in agreement with recent experimental observations /17/, opposes the previous conjecture /20/ that impurity transport ought to approach the neoclassical limit at high densities and lead to accumulation at the center of the discharge. Other theoretical results verified by the observations are the independence of the impurity confinement time from their charge to mass ratio $/ 18 /$ and 
its increase with the background ion mass: ${ }^{\tau} \mathrm{I} \sim \mathrm{m}_{i}^{\mathrm{s}}, 5 / 8<s<17 / 8$. By comparison we find $\tau_{i} \sim m_{i}^{5 / 8}$ which fits well with the Hugil1-Sheffield scaling law $/ 21 /$.

The importance of impurity transport studies is linked to their radiation losses, to the confinement of the fusion products, and to the consequent dilution of the reacting ions. The threat that the impurity accumulation which is predicted by classical /22/ and neoclassical /23/ theories represents for the tapping of thermonuclear energy resources has motivated the construction of specific experimental facilities to test means of reducing wal1-plasma contamination, to compare various wa11/1imiter materials, and more generally to study impurity behaviour more in depth. The present work is thus part of the theoretical programme supporting the TEXTOR experiment (Toroidal Experiment for Technology Oriented Research).

The paper is organized as follows. The technical details of the theory of anomalous impurity transport are developed in Section II. The rigorous results thus obtained are brought to a numerically tractable form in Section III. Expected impurity transport, including the role of sawtooth relaxations, and scaling laws with base ion mass are discussed in Section IV for two specific TFR discharges (Tokamak Fontenay-aux-Roses) and compared with experimental results. We briefly summarize our conclusions in Section $V$. 


\section{THEORY OF ANOMALOUS IMPURITY TRANSPORT}

The nonlinear drift kinetic equation $/ 3 /$ is readily solved for the distribution function of trace impurities (i.e. their space charge is negligible) and the density obtained, assuming weak turbulence, as a series $\mathrm{N}_{\mathrm{I}}=\mathrm{n}_{\mathrm{I}}^{(0)}+\mathrm{n}_{\mathrm{I}}^{(1)}+\mathrm{n}_{\mathrm{I}}^{(2)}$ where

$$
\begin{aligned}
& \mathrm{n}_{\mathrm{I}, \ell, \mathrm{m}}^{(0)}=-\frac{\mathrm{q}_{\mathrm{I}}}{\mathrm{T}_{\mathrm{I}}} \Phi_{\ell, \mathrm{m}}^{(0)} \mathrm{x}_{\mathrm{I}}(\overrightarrow{\mathrm{k}}, \omega), \\
& \mathrm{n}_{\mathrm{I}, \ell, \mathrm{m}}^{(1)}=-\frac{\mathrm{q}_{\mathrm{I}}}{\mathrm{T}_{\mathrm{I}}}\left[\Phi_{\ell, \mathrm{m}}^{(1)} x_{\mathrm{I}}(\overrightarrow{\mathrm{k}}, \omega)-\frac{\mathrm{i}}{2} \frac{\mathrm{c}}{\mathrm{B}} \sum_{\ell^{\prime}, \mathrm{m}^{\prime}} \Phi_{\ell^{\prime}, \mathrm{m}^{\prime}}^{(0)} \Phi_{\ell^{\prime \prime}, \mathrm{m}^{\prime \prime}}^{(0)} \mathrm{v}_{\mathrm{I}}\left(\overrightarrow{\mathrm{k}}, \omega \mid \overrightarrow{\mathrm{k}}^{\prime \prime}, \omega^{\prime \prime}\right)\right], \\
& \mathrm{n}_{I, \ell, m}^{(2)}=-\frac{\mathrm{q}_{I}}{\mathrm{~T}_{\mathrm{I}}}\left\{\Phi_{\ell, \mathrm{m}}^{(2)} x_{I}(\overrightarrow{\mathrm{k}}, \omega)+\mathrm{i} \frac{\mathrm{d}}{\mathrm{dt}}\left[\frac{\partial x_{I}(\vec{k}, \omega)}{\partial \omega} \Phi_{\ell, \mathrm{m}}^{(0)}\right\}\right. \\
& +\Phi_{\ell, m}^{(0)} \frac{c^{2}}{B^{2}} \sum_{\ell}^{\prime}, m^{\prime}\left[\frac{v_{i}\left(\vec{k}^{\prime \prime}, \omega^{\prime \prime} \mid \vec{k}, \omega\right)}{\varepsilon\left(\vec{k}^{\prime \prime}, \omega^{\prime \prime}\right)} v_{I}\left(\vec{k}, \omega \mid \vec{k}^{\prime \prime}, \omega^{\prime \prime}\right)\right. \\
& \left.\left.-w_{I}\left(\vec{k}, \omega|| \vec{k}^{\prime \prime}, \omega^{\prime \prime} \mid \vec{k}, \omega\right)\right]\left|\Phi_{\ell}^{(0), m^{\prime}}\right|^{2}\right\}
\end{aligned}
$$

The total derivative is defined by

$$
\begin{aligned}
\frac{\mathrm{d}}{\mathrm{dt}}\left[\frac{\partial \mathrm{X}}{\partial \omega} \Phi^{(0)}\right] & =\left(\frac{\partial}{\partial t}+\frac{\partial}{\partial \mathrm{r}} \mathrm{v}_{\mathrm{g}}\right)\left[\frac{\partial \chi}{\partial \omega} \Phi^{(0)}\right] \\
& =\frac{1}{2} \Phi^{(0)} \frac{\mathrm{d}}{\mathrm{dt}} \frac{\partial \chi}{\partial \omega}+\frac{\partial \chi}{\partial \omega} \frac{\mathrm{d} \Phi}{\mathrm{dt}} .
\end{aligned}
$$

$v_{g}=-\left(\partial \varepsilon / \partial k_{r}\right) /(\partial \varepsilon / \partial \omega)$ is the radial group velocity; the factor $1 / 2$ is quite common in the perturbation theory of quantum mechanical systems. In these expressions, the electrostatic potential $\Phi_{l, m}^{(0)}=\Phi_{l, m}^{(0)}(r-r, l, m, t)$ is the slowly varying amplitude (in the context of multiple time and length scales, see e.g. /24/) of the W.K.B. eigenmode 


$$
\Phi_{\ell, m}^{(0)}\left(r-r_{\ell, m}, t\right) \exp \left[i \int_{0}^{r-r} k_{r}(x) d x-i \omega t\right]
$$

with toroidal $(\phi)$ and poloidal $(\theta)$ mode numbers $\ell$ and $m$. These define the rationa1 surface $r_{\ell, m}$ on which $\vec{k} \cdot \vec{B}=0$ via the equation $\ell q\left(r_{\ell, m}\right)+m=0$ where $q=r B_{\phi} / R B_{\theta}$ is the safety factor. The wave eigenenergy $\omega$ and eigenmomentum $k_{r}$ obtain from the dispersion equation

$$
\begin{aligned}
\varepsilon(\vec{k}, \omega) & \equiv \tau+x_{i}(\vec{k}, \omega) . \\
& \equiv \tau+1-\int d v \frac{\omega-\omega_{i, \vec{k}}^{*, T}}{-k_{\|} \|_{\|}} J_{O, \vec{k}}^{2} F_{i}^{M}
\end{aligned}
$$

where $\chi_{i}$ is the base ion susceptibility, $k_{\|}\left[=\vec{k} \cdot \vec{B} / B=\left(\ell B_{\phi} / R B\right)\right.$ (denq/dr) $\left.\left(\mathrm{r}-\mathrm{r}_{\ell, \mathrm{m}}\right)=-\left(\mathrm{m} / \mathrm{rL}_{\mathrm{s}}\right)\left(\mathrm{r}-\mathrm{r}_{\ell, \mathrm{m}}\right)\right]$ is the parallel mode number, $\omega_{i, \vec{k}}^{\star}[=$ - $\left(\ell \mathrm{cT}_{i} / \mathrm{q}_{i} \mathrm{RB}_{\theta}\right) \mathrm{d} \ell \mathrm{nN} / \mathrm{dr} \mathrm{j}$ is the ion diamagnetic frequency, $\mathrm{J}_{0, \vec{k}}=\mathrm{J}_{0}\left(\mathrm{k}_{\perp} \mathrm{v}_{-} / \Omega_{i}\right)$ is the Bessel function of the first kind, $\Omega_{i}$ is the Larmor frequency, and $\tau=\mathrm{T}_{\mathrm{i}} / \mathrm{T}_{\mathrm{e}}$. In the cold plasma approximation $\mathrm{Eq}$. (3a) reduces to

$$
\varepsilon(\vec{k}, \omega)=\tau\left[1-\frac{\omega_{e, \vec{k}}^{t}}{\omega}+\frac{k_{\perp}^{2} c_{s}^{2}}{\Omega_{i}^{2}}-\frac{k^{2} c_{s}^{2}}{\omega^{2}}\right]=0
$$

from which there results that $\omega=\omega_{e, k}^{*} /\left(1+k_{\theta}^{2} a_{s}^{2}\right)$ and $k_{r}=-\left(\Omega_{i} / \omega\right)|k$, $\left(\mathrm{r}-\mathrm{r}_{\ell, \mathrm{m}}\right)\left[\mathrm{c}_{\mathrm{s}}=\left(\mathrm{T}_{\mathrm{e}} / \mathrm{m}_{\mathrm{i}}\right)^{\frac{1}{2}}\right.$ is the sound speed; $\mathrm{a}_{\mathrm{s}}=\mathrm{c}_{\mathrm{s}} / \Omega_{\mathrm{i}}$ is the ion sound Larmor radius; $\mathrm{k} \dot{ }=\partial \mathrm{k} \|^{/ \partial \mathrm{r}}$ and the choice of sign follows from causality; $\mathrm{k}_{\theta}=\mathrm{m} / \mathrm{r}$. Returning to Eq. (1) we note the definitions $\overrightarrow{\mathrm{k}}^{\prime}+\overrightarrow{\mathrm{k}}^{\prime \prime}=\overrightarrow{\mathrm{k}}$ and $\omega^{\prime}+\omega^{\prime \prime}=\omega$ and the conservation $1 \mathrm{aw}$

$$
\int_{0}^{r-r} \ell^{\prime}, m^{\prime} k_{r}^{\prime} d x+\int_{0}^{r-r} \ell^{\prime \prime}, m^{\prime \prime} k_{r}^{\prime \prime} d x=\int_{0}^{r-r} \ell, m k_{r} d x
$$

which follows from $\ell^{\prime} r_{\ell^{\prime}, m^{\prime}}+\ell^{\prime \prime} r_{\ell^{\prime \prime}, m^{\prime \prime}}=\ell r_{\ell, m}$. The radial mode number and the frequency of a beat wave (e.g. of toroidal mode number $l^{\prime \prime}$ and poloidal 
mode number $\mathrm{m} "$ ) are however no eigenmomentum nor eigenenergy; that is to say

$$
k_{r}-k_{r}^{\prime} \neq-\frac{\Omega_{i}\left|k^{\prime \prime}\right|\left(r-r_{\ell^{\prime \prime}, m^{\prime \prime}}\right)}{\omega_{e, \vec{k}^{\prime \prime}}^{*} /\left(1+k_{\theta}^{\prime \prime 2} a_{s}^{2}\right)} ; \quad \omega-\omega^{\prime} \neq \frac{\omega_{e, k^{\prime \prime}}^{*}}{1+k_{\theta}^{\prime 2} a_{s}^{2}}
$$

The beat wave $\Phi \vec{k}(1)$ obtained self consistently from the charge neutrality condition writes /3/:

$$
\Phi \frac{(1)}{\mathrm{k}}=\frac{i}{2} \varepsilon^{-1}(\mathrm{k}, \omega) \quad \frac{\mathrm{c}}{\mathrm{B}} \sum_{\ell^{\prime}, \mathrm{m}^{\prime}} \underset{\Phi_{\overrightarrow{\mathrm{k}}}^{\prime}}{(0)} \Phi_{\overrightarrow{\mathrm{k}}^{\prime \prime}}^{(0)} \mathrm{v}_{i}\left(\overrightarrow{\mathrm{k}}, \omega \mid \overrightarrow{\mathrm{k}}^{\prime \prime}, \omega^{\prime \prime}\right)
$$

(hence $\mathrm{n}_{\mathrm{i}}^{(1)}=-\mathrm{q}_{\mathrm{e}} \Phi^{(1)} / \mathrm{T}_{\mathrm{e}}=\mathrm{n}_{\mathrm{e}}^{(1)}$ ) whilst the definition of the matrix elements is as follows:

$$
\begin{aligned}
& v_{J}\left(\vec{k}, \omega \mid \vec{k}^{\prime \prime}, \omega^{\prime \prime}\right)=v_{J}\left(\vec{k}^{\prime \prime}, \omega \mid \vec{k}^{\prime}, \omega^{\prime}\right)
\end{aligned}
$$

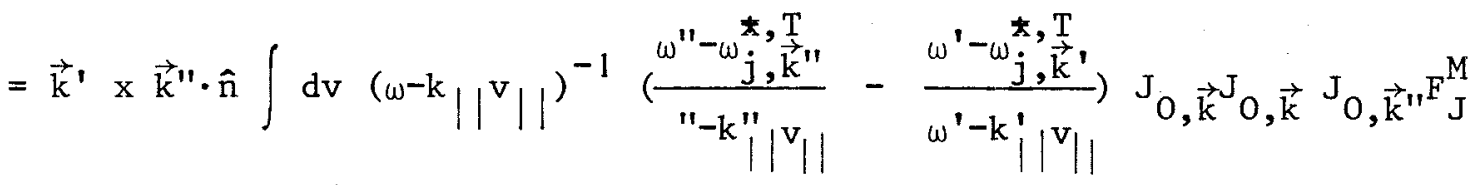

and $\quad w_{J}\left(\vec{k}, \omega|| \vec{k}^{\prime \prime}, \omega^{\prime \prime} \mid k, \omega\right)$

$$
\begin{aligned}
& =\left(\vec{k} \times \vec{k}^{\prime} \cdot \hat{n}\right)^{2} \int \mathrm{dv}(\omega-\mathrm{k}\|\mathrm{v}\|)^{-1}\left(\omega^{\prime \prime}-\mathrm{k}\|\|^{v} \|\right)^{-1}
\end{aligned}
$$

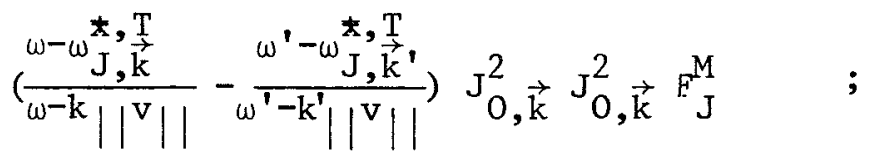

note the symmetry of the $v^{\prime} s$ with respect to the interchange of the last set of variables, e.g. $\left(\vec{k}^{\prime \prime}, \omega^{\prime \prime}\right)$, and the difference between the first and the last sets, e.g. $(\vec{k}, \omega)-\left(\vec{k}^{\prime \prime}, \omega^{\prime \prime}\right)=\left(\vec{k}^{\prime}, \omega^{\prime}\right)$.

Still the second and third terms of Eq. (1c) arise from wave energy losses through radiation (which leads to shear damping); the fourth term vanishes for steady state saturated turbulence. 
The formal expressions for the impurity flux $\Gamma_{I}$ and diffusion coefficient $D_{I}$ are now easily derived from the definition equations (see $/ 10 /)$

$$
\begin{aligned}
\frac{\partial N_{I}}{\partial t} & =\frac{1}{r} \frac{\partial}{\partial r} r \Gamma_{I}=-\frac{1}{r} \frac{\partial}{\partial r} r D_{I} \frac{\partial}{\partial r} N_{I} \\
& =\frac{-1}{r} \frac{\partial}{\partial r} \frac{c}{B} \sum_{\ell, m} i m \underset{\vec{k}}{\stackrel{\hbar}{s}}\left(r-r_{\ell, m}\right) n_{\vec{k}}\left(r-r_{\ell, m}\right) .
\end{aligned}
$$

The sum is made up of two contributions:

$$
\begin{aligned}
& \sum_{\ell, \mathrm{m}} \mathrm{i} \operatorname{m} \underset{\overrightarrow{\mathrm{k}}}{\stackrel{(1)^{*}}{*}} \mathrm{n}_{\overrightarrow{\mathrm{k}}}^{(1)} \\
& =-\left.\left.\left.\left.\frac{1}{2} \frac{\mathrm{q}_{\mathrm{I}}}{\mathrm{T}_{\mathrm{I}}} \frac{\mathrm{c}^{2}}{\mathrm{~B}^{2}} \sum_{\ell, \mathrm{m}} \sum_{\ell^{\prime}, \mathrm{m}^{\prime}} \mathrm{i} \mathrm{m}^{\prime \prime}\right|_{\overrightarrow{\mathrm{k}}} ^{(0)}\right|^{2}\right|_{\overrightarrow{\mathrm{k}}^{\prime}} ^{(0)}\right|^{2} \frac{\left|\mathrm{v}_{\mathrm{i}}\left(\overrightarrow{\mathrm{k}}^{\prime \prime}, \omega^{\prime \prime} \mid \overrightarrow{\mathrm{k}}, \omega\right)\right|^{2}}{\left|\varepsilon\left(\overrightarrow{\mathrm{k}}^{\prime \prime}, \omega^{\prime \prime}\right)\right|^{2}} \\
& \left.x_{I}\left(\vec{k}^{\prime \prime}, \omega^{\prime \prime}\right)-\frac{v_{i}^{\star}\left(\vec{k}^{\prime \prime}, \omega^{\prime \prime} \mid \vec{k}, \omega\right)}{\varepsilon^{\star}\left(\vec{k}^{\prime \prime}, \omega^{\prime \prime}\right)} v_{I}\left(\vec{k}^{\prime \prime}, \omega^{\prime \prime} \mid \vec{k}, \omega\right)\right]
\end{aligned}
$$

(we have first introduced the random phase approximation in the toroidal and poloidal variables $/ 25,26 /$ and then the substitution $\vec{k}, \vec{k}^{\prime}, \vec{k}^{\prime \prime} \leftrightarrow$ $\left.\vec{k}^{\prime \prime},-\vec{k}^{\prime}, \vec{k}\right)$ and

$$
\begin{aligned}
& \sum_{\ell, m} i m\left(\underset{\vec{k}}{(2) *} \mathrm{n}_{\overrightarrow{\mathrm{k}}}^{(0)}+\underset{\overrightarrow{\mathrm{k}}}{\left.(0) * \mathrm{n}_{\overrightarrow{\mathrm{k}}}^{(2)}\right)}\right.
\end{aligned}
$$

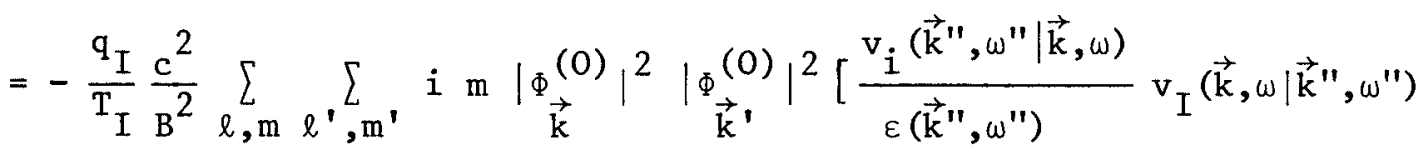

$$
\begin{aligned}
& \left.-w_{I}\left(\vec{k}, \omega|| \vec{k}^{\prime \prime}, \omega^{\prime \prime} \mid \vec{k}, \omega\right)\right] \\
& +\frac{1}{2} \frac{\mathrm{q}_{I}}{\mathrm{~T}_{\mathrm{I}}} \sum_{\ell, \mathrm{m}} \mathrm{m}\left\{\frac{\partial}{\partial \mathrm{t}}\left[\frac{\partial \mathrm{x}_{\mathrm{I}}(\mathrm{k}, \omega)}{\partial \omega}\left|\Phi_{\overrightarrow{\mathrm{k}}}^{(0)}\right|^{2}\right]-\frac{\partial}{\partial \mathrm{r}}\left[\frac{\partial \mathrm{x}_{\mathrm{I}}(\mathrm{k}, \omega)}{\partial \mathrm{k}_{\mathrm{r}}}\left|\Phi_{\mathrm{k}}^{(0)}\right|^{2}\right]\right\}
\end{aligned}
$$


Here we have taken $\operatorname{Imx}_{I}(\vec{k}, \omega)=0$ which is consistent with the cold plasma approximation for the linear eigenmodes (see Eq. (3b)). Before recombining (7a) and (7b), it is useful to look at the parity of the matrix elements (5a) and (5b) with respect to various interchanges of variables. Firstly, if $\left(\vec{k}, \vec{k}^{\prime}, \vec{k}^{\prime \prime}\right) \rightarrow\left(\vec{k}^{\prime \prime},-\vec{k}^{\prime}, \vec{k}\right)$, then

$$
v\left(\vec{k}, \omega \mid \vec{k}^{\prime \prime}, \omega^{\prime \prime}\right)=-v\left(\vec{k}^{\prime \prime}, \omega^{\prime \prime} \mid \vec{k}, \omega\right)
$$

this result holds because the contribution from the pôle where $v_{\|}=\omega^{\prime} / k||$ is neglected (the wave $\vec{k}^{\prime}, \omega^{\prime}$ is a linear eigenmode). Secondly, if $\left(\vec{k}, \vec{k}^{\prime}, \vec{k}^{\prime \prime}\right) \rightarrow\left(\vec{k}^{\prime}, \vec{k},-\vec{k}^{\prime \prime}\right)$, then

$$
\mathrm{v}\left(\overrightarrow{\mathrm{k}}^{\prime \prime}, \omega^{\prime \prime} \mid \overrightarrow{\mathrm{k}}, \omega\right)=-\mathrm{v}^{\star}\left(-\overrightarrow{\mathrm{k}}^{\prime \prime},-\omega^{\prime \prime} \mid \overrightarrow{\mathrm{k}}^{\prime}, \omega^{\prime}\right)=-\mathrm{v}^{\star}\left(-\overrightarrow{\mathrm{k}}^{\prime \prime},-\omega^{\prime \prime} \mid-\overrightarrow{\mathrm{k}},-\omega\right)
$$

is the consequence of the uniqueness of the Landau continuation:

$$
\begin{aligned}
& \left(\omega^{\prime \prime}-\left.k^{\prime \prime}\right|^{v}||^{+i \lambda}\right) \rightarrow\left(-\omega^{\prime \prime}+\left.k^{\prime \prime}\right|^{v}||^{+i \lambda}\right), \lambda \rightarrow 0^{+} \text {. Defining } w=w^{+}+w^{-} \text {with } \\
& \quad w^{ \pm}\left(\vec{k}, \omega|| \vec{k} ", \omega^{\prime \prime} \mid \vec{k}, \omega\right)=\frac{1}{2}\left[w\left(\vec{k}, \omega|| \vec{k}^{\prime \prime}, \omega^{\prime \prime} \mid k, \omega\right) \pm w\left(\vec{k},, \omega^{\prime}|| \vec{k}^{\prime \prime}, \omega^{\prime \prime} \mid k, \omega\right)\right]
\end{aligned}
$$

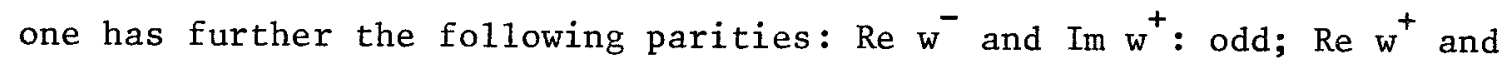
$\operatorname{Im} \mathrm{w}^{-}$: even.

These considerations lead to a somewhat simplified expression for the impurity flux:

$$
\begin{aligned}
& r \Gamma_{I}=\frac{1}{2} \frac{\mathrm{q}_{I}}{\mathrm{~T}_{I}} \frac{\mathrm{c}}{\mathrm{B}} \sum_{\ell, m} \mathrm{~m}\left\{-\frac{\partial}{\partial t}\left[\frac{\partial \mathrm{x}_{\mathrm{I}}(\overrightarrow{\mathrm{k}}, \omega)}{\partial \omega}\left|\Phi_{\vec{k}}\right|^{2}\right]+\frac{\partial}{\partial \mathrm{r}}\left[\frac{\partial \mathrm{X}_{\mathrm{I}}(\overrightarrow{\mathrm{k}}, \omega)}{\partial \mathrm{k}_{\mathrm{r}}}\left|\Phi_{\vec{k}}\right|^{2}\right]\right\} \\
& +\frac{1}{2} \frac{\mathrm{q}_{\mathrm{I}}}{\mathrm{T}_{\mathrm{I}}} \frac{\mathrm{c}^{3}}{\mathrm{~B}^{3}} \sum_{\ell, \mathrm{m} \ell^{\prime}, \mathrm{m}^{\prime}} \mathrm{m}^{\prime \prime}\left|\Phi_{\vec{k}}\right|^{2}\left|\Phi_{\vec{k}},\right|^{2}\left\{\frac{\left|\mathrm{v}_{\mathrm{i}}\left(\overrightarrow{\mathrm{k}}^{\prime \prime}, \omega^{\prime \prime} \mid \overrightarrow{\mathrm{k}}, \omega\right)\right|^{2}}{\left|\varepsilon\left(\overrightarrow{\mathrm{k}}^{\prime \prime}, \omega^{\prime \prime}\right)\right|^{2}} \operatorname{Im} x_{\mathrm{I}}\left(\overrightarrow{\mathrm{k}}^{\prime \prime}, \omega^{\prime \prime}\right)\right.
\end{aligned}
$$




$$
\begin{aligned}
& \left.-\left[\frac{v_{i}\left(\vec{k}^{\prime \prime}, \omega^{\prime \prime} \mid \vec{k}, \omega\right)}{\varepsilon\left(\vec{k}^{\prime \prime}, \omega^{\prime \prime}\right)}+c . c .\right] \operatorname{Im} v_{I}\left(\vec{k}^{\prime \prime}, \omega^{\prime \prime} \mid k, \omega\right)-\operatorname{Im}_{I}^{+}\left(\vec{k}, \omega|| \vec{k} ", \omega^{\prime \prime} \mid \vec{k}, \omega\right)\right\} \\
& -\frac{1}{2} \frac{\mathrm{q}_{I}}{T_{I}} \frac{c^{3}}{B^{3}} \sum_{\ell, m} \sum_{\ell^{\prime}, m^{\prime}}\left(m+m^{\prime}\right)\left|\Phi_{\vec{k}}\right|^{2}\left|\Phi_{\vec{k}}\right|^{2} \operatorname{Im} w_{I}^{-}\left(\vec{k}, \omega|| \vec{k} ", \omega^{\prime \prime} \mid \vec{k}, \omega\right) .
\end{aligned}
$$

We have split $\mathrm{m}=(1 / 2)\left[\mathrm{m}^{\prime \prime}+\left(\mathrm{m}-\mathrm{m}^{\prime}\right)\right\}$ in Eq. (7b), and dropped the superscript (0); c.c. is the complex conjugate. Eq. (8) demonstrates a result which is intuitively obvious: the transport of impurities arises solely from their resonant interaction with the waves; note also that the combination $v_{i}\left(\vec{k}^{\prime \prime}, \omega^{\prime \prime} \mid \vec{k}, \omega\right) / \varepsilon\left(\vec{k}^{\prime \prime}, \omega^{\prime \prime}\right)$ originates from the expression of the potential Eq. (5). If the test particle (I) identifies with the base ion (i), the first and third ("c.c") terms cancel out in the curly bracket; the other terms, including the linear ones, can be regrouped according to the wave

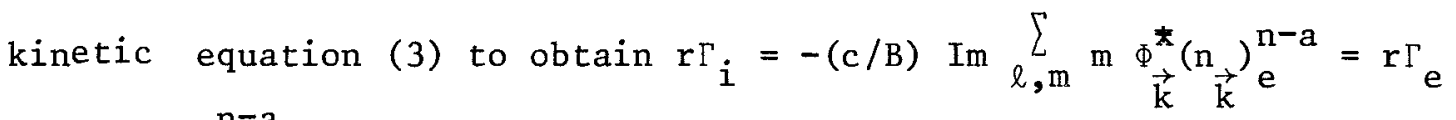
where $\left(\mathrm{n}_{\vec{k}}\right)^{\mathrm{n}-\mathrm{a}}$ is the out of phase, non-adiabatic electron response. Hence the ambipolar character of the transport. 
II. NUMERICAL ESTIMATES OF THE IMPURITY FLUXES

Light impurities are fully stripped over much of the plasma cross section. It is therefore extremely difficult to measure their density profiles and accurate experimental information with which to compare is lacking. Heavy impurities in their lower ionization states are confined to the plasma periphery where the proximity of mechanical parts obscures our understanding. For these reasons our theoretical studies are limited for the present to impurity ions of high atomic mass $m_{I}$ and high electric charge $\mathrm{q}_{\mathrm{I}}=\mathrm{Ze}$. More precisely we shall assume that the ratio of the Larmor radii

$$
a_{I}^{2} / a_{i}^{2}=\frac{\left(m_{I} / m_{i}\right)}{z^{2}} \frac{T_{I}}{T_{i}} \ll 1
$$

Hence $\operatorname{Im} \mathrm{w}_{I}^{-}=0$ whilst to lowest significant order, the expression in the curly bracket becomes

$$
\left|\frac{v_{i}\left(\vec{k}^{\prime \prime}, \omega^{\prime \prime} \mid \vec{k}, \omega\right)}{\varepsilon\left(\vec{k}^{\prime \prime}, \omega^{\prime \prime}\right)}-\frac{\left(\vec{k}^{\prime} \times \vec{k} \cdot \hat{n}\right) k^{\prime \prime} \mid}{k||^{\omega^{\prime}-k}||^{\omega}}+0\left(z^{-1}\right)\right|^{2} \operatorname{Im} x_{I}\left(\vec{k}^{\prime \prime}, \omega^{\prime \prime}\right) .
$$

We note that

$$
\begin{aligned}
& v_{i}\left(\vec{k}^{\prime \prime}, \omega^{\prime \prime} \mid \vec{k}, \omega\right)=-\frac{\vec{k}^{\prime} \times \vec{k} \cdot \hat{n}}{k \|\left.^{\omega^{\prime}-k}\right|^{\omega}} \int d v\left(k^{\prime \prime} \frac{\omega^{\prime \prime}-\omega_{i}^{*} \vec{k}^{\prime \prime}}{\omega^{\prime \prime}-k^{\prime \prime}\left\|^{v}\right\|}-\vec{k}^{\prime \prime} \leftrightarrow \vec{k}+\vec{k}^{\prime \prime} \leftrightarrow \vec{k}^{\prime}\right) \\
& J_{\circ, \vec{k}} J_{o \vec{k}}, J_{o k^{\prime \prime}} \\
& =\frac{\vec{k}^{\prime} \times \vec{k} \cdot \hat{n}}{k_{\mid} \omega^{\prime}-\left.k_{\mid}^{\prime}\right|^{\omega}}\left[k^{\prime \prime}|| \varepsilon\left(\vec{k}^{\prime \prime}, \omega^{\prime \prime}\right)-\int d v k^{\prime \prime} \frac{\omega^{\prime \prime}-\omega_{i, \vec{k}^{\prime \prime}}^{\star T}}{\omega^{\prime \prime}-\left.k^{\prime \prime}\right|^{v}||}\right.
\end{aligned}
$$

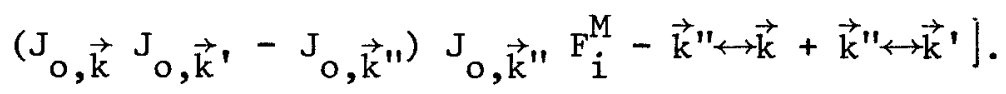

this last result being exact. The above considerations thus yield - correct 
up to order $z^{-1}-$ the flux

$$
\begin{aligned}
& r \Gamma_{I}=\frac{1}{2} \frac{q_{I}}{\dot{T}_{I}} \frac{c^{3}}{B^{3}} \sum_{\ell, m} \ell^{\prime}, m^{\prime} m^{\prime \prime}\left|\Phi_{\vec{k}}\right|^{2}\left|\Phi_{\vec{k}}\right|^{2} \frac{\left(\vec{k}^{\prime} x \vec{k} \cdot \hat{n}\right)^{2}}{\left(k||^{\prime}-k||^{\prime}\right)^{2}} \text { Im } x_{I}\left(\vec{k}^{\prime \prime}, \omega^{\prime \prime}\right)
\end{aligned}
$$

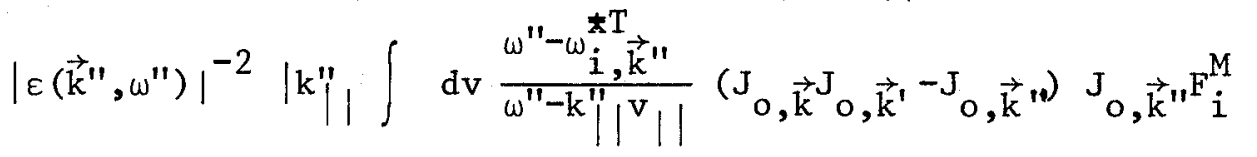

$$
\begin{aligned}
& -\vec{k}^{\prime \prime} \leftrightarrow \vec{k}+\left.\vec{k}^{\prime \prime} \leftrightarrow \vec{k}^{\prime}\right|^{2}
\end{aligned}
$$

in steady state conditions. Later in this Section, this result is further transformed with the help of the substitution

$$
\sum_{\ell}(\delta r)^{-1} \int_{r_{\ell, m}-\delta r / 2}^{r} d r\left|\Phi_{\vec{k}}^{0}\left(r-r_{\ell, m}\right)\right|^{2}=\left.\left.\Delta_{m}^{-1} \int_{-\infty}^{\infty} d x\right|_{\vec{k}} ^{(0)}(x, r)\right|^{2}
$$

which is a consequence of $\delta \mathrm{r} / \Delta_{\mathrm{m}}$ rational surfaces lying in the interval $\delta \mathrm{r}$. The latter is intermediate between the macro and the micro length scales which are respectively described by the variables $r$ and $x$. The toroidal mode number $\ell \cong-\mathrm{m} / \mathrm{q}(\mathrm{r})$ is fixed in Eq. (11) where $\Delta_{\mathrm{m}}=\left|\mathrm{mdq}^{-1} / \mathrm{dr}\right|^{-1}$ is the distance between the neighbouring rational surfaces $r_{\ell+1, m}$ and $r_{\ell, m}$. The susceptibility of the trace impurities is made up of two terms:

$$
\operatorname{Im} x_{I}\left(\vec{k}^{\prime \prime}, \omega^{\prime \prime}\right)=\sqrt{\pi} \frac{\omega^{\prime \prime}-\omega_{I}^{\star}, \vec{k}^{\prime \prime}}{\sqrt{2}\left|k_{\mid}^{\prime \prime}\right| c_{I}} \exp \left(-\omega^{\prime \prime 2} /\left.2 k^{\prime \prime}\right|^{2} c_{I}^{2}\right)
$$

where $c_{I}=\left(T_{I} / m_{I}\right)^{\frac{1}{2}}$ is the thermal speed. We shall neglect temperature gradients compared to density gradients, hence $\omega_{I}^{* T}=\omega_{I}^{\star}$. The first term gives rise to the anomalous frictional flux which is proportional to the base ion density gradient $\left(\omega^{\prime \prime}=\omega^{-} \omega^{\prime} \sim \mathrm{d} \ell \mathrm{nN}_{\mathbf{i}} / \mathrm{dr}\right)$. The second term yields the diffusional flux, proportional to the density gradient of the impurities $\left(\omega_{I, \vec{k}^{\prime \prime}}^{\star} \sim d \ln N_{I} / d r\right)$. 


\section{A. The Diffusional Flux}

Nonlocal scattering obviously provides here the dominant contribution. The poloidal mode numbers $k_{\theta}^{\prime}$ contributing effectively to transport are limited by the susceptibility (12) to a narrow range defined by

$$
\left(\mathrm{k}_{\theta}^{\prime}-\left\langle\mathrm{k}_{\theta}^{\prime}>\right)^{2} \equiv\left(\frac{\omega^{\prime \prime}}{\partial\left\langle\omega^{\prime}>/ \partial<k_{\theta}^{\prime}\right\rangle}\right)^{2} \leqslant \alpha^{2}\left|\frac{\sqrt{2}<k^{\prime \prime} \mid c_{I}}{\partial\left\langle\omega^{\prime}>/ \partial\left\langle k_{\theta}^{\prime}>\right.\right.}\right|^{2}<\alpha^{2} \frac{c_{I}^{2}}{c_{i}^{2}}<k_{\theta}^{\prime}>^{2}\right.
$$

where $\alpha \gtrsim 1$. In the cold plasma approximation, the central mode number is $\left\langle k_{\theta}^{\prime}\right\rangle \equiv 1 / k_{\theta} a_{s}^{2}$. The parallel mode number $\langle k| p=-\left\langle k_{\theta}^{\prime}\right\rangle x / L_{s}$, where, in estimating the orderof magnitude of $k \|_{1}^{(1)}, x$ is taken as the cut-off distance of the radial eigenfunction (see Ref. /10/, Sec. II C). Finally the last inequality follows from ion Landau damping of the linear modes being negligible $\left(\left.\sqrt{2} k_{1}\right|_{i}<\omega\right)$. To obtain explicitely the diffusive impurity flux, we can use the substitution (11) together with

$$
\sum_{\mathrm{m}} \sum_{\mathrm{m}^{\prime}}=\mathrm{r}^{2} \int_{-\infty}^{\infty} \mathrm{dk} \theta\left|\partial\left\langle\omega^{\prime}\right\rangle / \partial\left\langle\mathrm{k}_{\theta}^{\prime}\right\rangle\right|^{-1} \int_{-\infty}^{\infty} \mathrm{d} \omega^{\prime \prime}
$$

Thus after integration over $\omega^{\prime \prime}$ :

$$
\begin{aligned}
& \left(\Gamma_{I}\right)_{d}=-\frac{\pi}{2} r^{2} \frac{\mathrm{dN}_{\mathrm{I}}}{\mathrm{dr}} \mathrm{a}_{\mathrm{s}}^{4} \mathrm{c}_{\mathrm{s}}^{4} \int \mathrm{dk}_{\theta}\left|\frac{\partial\left\langle\mathrm{k}_{\theta}^{\prime}\right\rangle}{\partial\left\langle\omega^{\prime}\right\rangle}\right|\left(\left\langle\mathrm{k}_{\theta}^{\prime \prime}\right\rangle\right)^{2} \int \frac{\mathrm{dx}}{\Delta_{\mathrm{m}}} \int \frac{\mathrm{dx}}{\Delta_{\left\langle\mathrm{m}^{\prime}\right\rangle}} .
\end{aligned}
$$

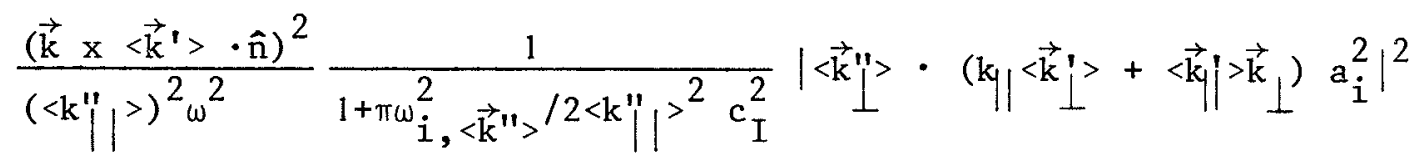

$$
\begin{aligned}
& \left|\frac{\mathrm{e}}{\mathrm{T}_{\mathrm{e}}} \Phi_{\overrightarrow{\mathrm{k}}}\right|^{2}\left|\frac{\mathrm{e}}{\mathrm{T}_{\mathrm{e}}} \Phi_{\left\langle\overrightarrow{\mathrm{k}}^{\prime}>\right.}\right|^{2}
\end{aligned}
$$

One recognizes easily in this result the Larmor radius expansion of the last factor in Eq. (10) and the dielectric function (see Appendix A)

$$
\varepsilon\left(\vec{k}^{\prime \prime}, \omega^{\prime \prime \rightarrow 0}\right)=1-i \sqrt{\pi} \omega_{i, \vec{k}^{\prime \prime}}^{\star} / \sqrt{2}\left|k^{\prime \prime}\right| \mid c_{i} .
$$


The expression used for numerical evaluation is obtained by expressing $k_{r}^{(')}$ and $k_{\|}^{(')}$ as functions of $x$ and $x^{\prime}$. Hence, with $\xi=\left(x \Omega_{i} x_{t}^{2} /\right.$ $\left.\mathrm{L}_{\mathrm{s}} \omega \mathrm{a}_{\mathrm{s}} \mathrm{x}_{\mathrm{c}}\right)$ and $\mathrm{y}=\mathrm{k}_{\theta}^{2} \mathrm{a}_{\mathrm{s}}^{2}$,

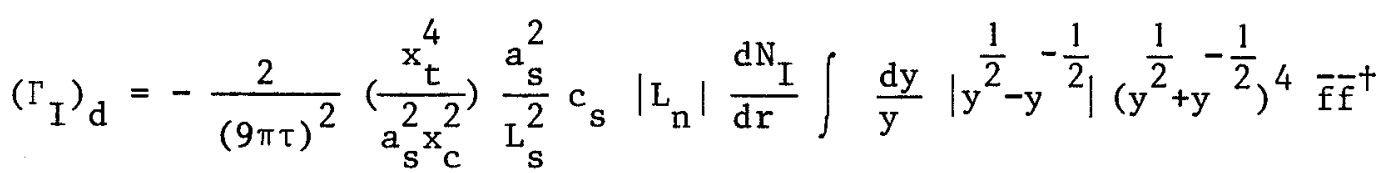

$$
\begin{aligned}
& \int \mathrm{d} \xi \int \mathrm{d} \xi^{\prime}\left[\left(\xi \mathrm{y}^{\frac{1}{2}}-\xi^{\prime} \mathrm{y}^{-\frac{1}{2}}\right)^{2}+\frac{\pi \tau}{2}\left(y-y^{-1}\right)^{2} \frac{\mathrm{x}_{t}^{4}}{a_{\mathrm{s}}^{2} \mathrm{x}_{c}^{2}}\right]^{-2}\left(\xi-\xi^{\prime}\right)^{2} \\
& x\left[\xi \xi^{\prime}\left(y^{\frac{1}{2}} \xi-y^{-\frac{1}{2}} \xi^{\prime}\right)+\frac{1}{2}\left(\xi+\xi^{\prime}\right)\left(y^{\frac{1}{2}}-y^{-\frac{1}{2}}\right)\right]^{2} \\
& \exp \left[-\left(\xi^{2} y+\xi^{\prime 2} y^{-1}\right)\right]
\end{aligned}
$$

The spectral functions $\bar{f}(y) \sim\left|e \Phi_{\vec{k}}(x=0) / T_{e}\right|^{2}$ (see Eq. (12b) of Ref./10/) and $\overline{\mathrm{f}}^{\dagger}(\mathrm{y}) \equiv \overline{\mathrm{f}}\left(\mathrm{y}^{-1}\right)$ are to be obtained from the wave kinetic equation (3)

$$
\left\{\left(y \frac{\partial}{\partial y}+\frac{2-y}{1-y}\right) \bar{f}+\left\{(2-y)^{2} \frac{\partial}{\partial y}+\frac{2-y}{1-y}\right] \bar{f}^{\dagger}\right\} \bar{f}=-\operatorname{sign}(1-y) \tilde{\gamma}_{L} \frac{(1-y)^{2}}{(1+y)^{3}} y^{-\frac{3}{2}} \bar{f}
$$

(the nonlinear character of this equation is linked to the constraint that the spectrum is positive definite: $f \geqslant 0$ ), where $\tilde{\gamma}_{L}$ is related to the linear growth rate: $\gamma_{L}=\tilde{\gamma}_{L}\left|\omega L_{n} / L_{s}\right|$. We have defined the Pearlstein-Berk turning point $x_{t}=a_{s}\left|L_{s} / L_{n}(1+y)\right|^{1 / 2}$ and the linear cut-off distance $x_{c}$ $\left[\left|\Phi_{\mathrm{k}}\right|^{2} \sim \exp \left(-\mathrm{x}^{2} / \mathrm{x}_{\mathrm{c}}^{2}\right)\right]$

A typical spectrum $\left|e_{\theta}, \vec{k}\right|^{2}=\left|k_{\theta}{ }^{\Phi} \vec{k}\right|^{2} \sim f(y)$ is shown in Fig. 1; it was obtained for the macroscopic parameters, at the normalized radius $\rho=0.5$, of a moderate density discharge in T.F.R. /11/. The impurity diffusion coefficient obtained from Eq. (14b) and the definition relation 
$\left(\Gamma_{I}\right)_{d}=-\left(D_{I}\right)_{d} \partial N_{I} / \partial r$ is given as a function of radius in Fig. 2; for comparison we show the anomalous diffusion coefficient of the base ions $\left(D_{i} \sim K_{e} / 5\right.$; the electron heat transport coefficient was calculated in $/ 10 /$ ) and the Rutherford neo-classical value in $/ 23 /$. Corresponding results are reported in Fig. 3 for a high density TFR discharge.

B. The Frictional F1ux

Numerically, local scattering provides the dominant contribution to the anomalous frictional flux. Thus $k_{\theta}^{\prime \prime}=\omega^{\prime \prime} /\left(\partial \omega / \partial \mathrm{k}_{\theta}\right) \ll \mathrm{k}_{\mathrm{r}}^{\prime \prime}$ and $\varepsilon\left(\vec{k}^{\prime \prime}, \omega^{\prime \prime} \rightarrow 0\right)=1$. Proceeding otherwise as in Sec. III A, one easi1y arrives at the result

$$
\begin{aligned}
\left(\Gamma_{I}\right)_{f}= & -\frac{2}{(9 \pi \tau)^{2}} \frac{q_{I} m_{i}}{q_{i} m_{I}} \frac{a_{s}^{2}}{L_{s}^{2}} c_{s} \frac{L_{n}}{T_{n} \mid} N_{I} \int \frac{d y}{1 / 2}\left[\frac{y^{2}(1+y)^{2}}{1-y} \bar{f}\right]^{2} \operatorname{sign}(1-y) \\
& \int d \xi \int d \xi^{\prime}\left(\xi-\xi^{\prime}\right)^{4} \xi^{2} \xi^{\prime 2} \exp \left[-\left(\xi^{2}+\xi^{\prime 2}\right) y\right] .
\end{aligned}
$$

The impurity friction coefficient obtained from Eq. (16) and the definition $\left(\Gamma_{I}\right)_{f}=-\left(D_{I}\right)_{f} N_{I} / L_{n}$ is compared in Fig. 4 to the corresponding neoclassical values in the moderate density TFR discharge of Sec. III A. The numerical calculation, where we have assumed $z=12$ and fully striped impurities $\left(\mathrm{q}_{\mathrm{I}} \mathrm{m}_{i} / \mathrm{q}_{i} \mathrm{~m}_{\mathrm{I}}=1\right.$ in this deuterium plasma), show that the anomalous contribution plays a marginal role. 


\section{DISCUSSION IF IMPURITY TRANSPORT}

The theoretical growth rate of the trapped electron mode is not unequivocally determined for various reasons: (i) out of mathematica1 convenience, e.g. calculation by perturbation, neglect of electron inertia $127 /, \ldots$, (ii) out of lack of experimental information, e.g. on the radial distribution of the effective charge and thus of the collisionality, ..., (iii) because the release of power in most machines has a cyclic pattern, due to the sawtooth relaxations of the core, which implies an adiabatic variation of $\gamma_{L}$. It is therefore not surprising that an empirical weight factor must be introduced in the expression of the linear growth rate in order to reproduce theoretically the average power balance anomaly (this empirical factor $=1$, respectively $=2$, for the moderate, respectively the high density discharges that we consider here). The anomalous impurity coefficients shown in Figs. 2, 3 and 4 are obtained with these adjusted rates. Though representative, they differ from the average impurity transport for the relation between the $D^{\prime} s$ and $\gamma_{L}$ is highly nonlinear, the difference being more pronounced at the higher densities where the role of the relaxations increases.

In the following, we discuss separately the transport without and during relaxations. We comment also on the scaling in regards to the base ion atomic mass.

A. Impurity transport in the absence of relaxations.

From the numerical results shown in Figs. 2, 3, and 4, we draw the following conclusions.

1. - The diffusion induced by the trapped electron instability is ineffective in the inner and in the outer plasma layers where other transport mechanisms must be called upon. Hereafter, "typical" of turbulent transport is the layer where $\mathrm{D}_{e}$ and $\mathrm{D}_{\mathrm{I}}$ maximize. 
2. - The anomalous diffusion coefficients of the trace impurities and of the base ions (or electrons) are quite similar although the profile of $\mathrm{D}_{\mathrm{I}}$ is slightly shifted towards the plasma edge. This numerical similarity is intriguing particularly in view of the very different pace of growth of $\mathrm{D}_{e}$ and $\mathrm{D}_{I}$ with increasing $\gamma_{L}$ (see Secticn IV B.).

3. - The Rutherford neoclassical diffusion coefficient /23/

$$
\left(D_{I}\right)_{d}^{n c}=2 q^{2} a_{s}^{2}\left(m_{e} T_{e} / m_{i} T_{i}\right)^{\frac{1}{2}} \tau_{e}^{-1}\left(1+n_{I}\right) T_{I} / T_{i}
$$

is, in the moderate density discharge, about a factor twelve (12) smaller than the corresponding anomalous coefficient $\left(D_{I}\right)_{d^{*}}\left(\tau_{e}\right.$ is the Braginskii collision time and, in the numerical estimates, $n_{I}=d \ln T_{I} / d \operatorname{lnN}=1=T_{I} / T_{i}$ ). The neoclassical frictional coefficient

$$
\left(D_{I}\right)_{f}^{n c}=2 z q^{2} a_{s}^{2}\left(m_{e} T_{e} / m_{i} T_{i}\right)^{\frac{1}{2}} \tau_{e}^{-1}\left(1+n_{i}\right)
$$

on the other hand exceeds its anomalous analogue when $z \geq 12$. These theoretical predictions agree well with the conclusions from an impurity injection experiment in a macroscopically similar TFR plasma /19/ and with results from Alcator $\mathrm{A} / 17 /$ and Alcator $\mathrm{C} / 18 /$.

4. - The ratios $\left(D_{I}\right)^{n c} /\left(D_{I}\right)$ are larger in the high density discharge; we emphasize that this result is not significative of the relative cleanness of high and moderate density plasmas because of the variation of sawteeth activity (see Section IV B. below). 
B. Effect of relaxations on impurity transport

If the destabilizing linear response of the trapped electrons corresponding to Sec. IV A is increased by the factor 1.5, we find that the base ion and the impurity diffusion coefficients increase in the ratios $>10$ and $>10^{2}$ respectively at the normalized radius $\rho=0.5$ of the moderate density discharge. This rapid variation of $\mathrm{D}_{e}$ has motivated us to propose $19,10 /$ that the heat bursts released by the relaxations are capable of enhancing sufficiently the conducting turbulence as to sustain their own transport: during these short events, the coefficients of electron heat conduction and diffusion must indeed increase by a factor of the order of the ratio of the rise and decay times of the sawteeth $(\simeq 30)$. The still faster variation of $D_{I}$ is consistent with other Alcator $C$ injection studies /28/ showing that the central density of peaked silicon profiles decreases by $\sim 50 \%$ during the $30 \mu \mathrm{sec}$ that lasts a relaxation. Still in agreement with the theory, the central density of hollow profiles (i.e. before the injected impurities have completed their inward motion), in contrast, increases very suddenly during a relaxation /29/. Finally, other experimental information indicates that intrinsic impurities move in and out during the sawtooth cycle /30/ and that there is a correlation of impurity confinement with magnetohydrodynamic activity /31/. Our theory provides an interpretation of this cleaning action of the sawteeth.

C. Scaling with base ion mass.

Convincing scaling laws for drift transport cannot be derived given the rapid variations of particle and energy fluxes with the linear growth rate. Indeed even small changes in the total current, mean particle density, or toroidal magnetic field, will inevitably tend to induce large modifications of the transport coefficients; these will alter the profiles (of current and particle densities) and hence feed back on the growth rate 
of the modes and on the transport (Generally speaking, scaling laws apply only if the profiles remain self-similar; this requires that the feed back be weak).

The theoretical results rather establish a link, according to the marginal instability condition $\gamma_{L} \rightarrow 0$, between the independent parameters entering the definition of the linear growth rate. Hence

$$
\mathscr{R}_{0}\left[\frac{\mathrm{q}_{\mathrm{a}}^{\mathrm{R}}}{\mathrm{a}}, \frac{\mathrm{q}_{\mathrm{a}} \mathrm{T}^{2}, \mathrm{o}^{/ \mathrm{N}_{\mathrm{o}}}}{(\mathrm{aR})^{\frac{1}{2}} \mathrm{~m}_{\mathrm{i}}^{\frac{1}{2}}\left(1+\mathrm{Z}_{\mathrm{eff}}\right)}\right]=\mathrm{k}
$$

(see Eq. (7b) of Ref. /10/) where the choice of the constant $k$ depends moderately on the temperature and density profiles and on the width of the turbulent plasma layer. We derived previously $/ 9,10 /$ from Eq. (18) a promising expression for the high density limit. We propose now to derive scaling laws, with respect to the base ion mass, for the anomalous electron heat conduction coefficient $\left(\mathrm{K}_{\mathrm{e}}\right)$, the electron diffusion coefficient $\left(D_{e} \sim \mathrm{K}_{\mathrm{e}} / 5\right.$ for the trapped electron instability) and the impurity diffusion coefficient. Only the temperature and the loop voltage are allowed to vary in order to compensate for the modifications of the ion mass.

Eq. (18) implies that $\mathrm{T}_{\mathrm{e}, \mathrm{o}} \sim \mathrm{m}_{i}^{1 / 4}$ whereas, from Ohm's law, the loop voltage $\mathrm{V} \sim \mathrm{T}_{\mathrm{e}, \mathrm{o}}^{-3 / 2} \sim \mathrm{m}_{\mathrm{i}}^{-3 / 8}$. The equation of macroscopic energy balance $\vec{J} \cdot \overrightarrow{\nabla V}=\vec{\nabla} \cdot K_{e} \overrightarrow{N \nabla T}$ e (consider $\mathrm{V}$ as a multivalued function) where we neglect radiation losses, etc. ..., and assume stationarity then yields

$$
K_{e} \sim D_{e} \sim m_{i}^{-5 / 8}
$$

which agrees well with the Hugill-Sheffield scaling $\tau_{E} \sim A_{i}^{0.8} / 21 /$. The microscopic expression of the heat conduction coefficient (Eq. (18b) of Ref. /10/) implies that 


$$
\mathrm{K}_{\mathrm{e}} \sim \mathrm{T}_{\mathrm{e}, \mathrm{o}}^{7 / 2} \mathrm{G}_{1} \sim \mathrm{T}_{\mathrm{e}, \mathrm{o}}^{7 / 2} \int_{0}^{\infty} \frac{\mathrm{dy}}{\mathrm{y}}(1+\mathrm{y}) \overline{\mathrm{f}}
$$

for self-similar profiles. Hence $G_{1} \sim \mathrm{m}_{i}^{-3 / 2} / 32 /$. If we assume that $\overline{\mathrm{f}}$ scales like $G_{1}$, and that the integral occuring in the definition (14b) of the impurity flux scales like $\overline{\mathrm{f}}^{2}$, then we find $\left(D_{I}\right)_{d} \sim m_{i}^{-17 / 8}$. This assumption is probably too severe; for example the impurity diffusion coefficient must reduce to $D_{e}$ when the trace impurity identifies with the base ion species. It is thus fair to consider that $\left(\Gamma_{I}\right)_{d} \sim T^{3 / 2} m_{i}^{1 / 2} G_{1}^{(1+\theta)}$ where $0<\theta<1$. Hence

$$
\left(D_{I}\right)_{d} \sim m_{i}^{-s}, \frac{5}{8} \leqslant s=\frac{5}{8}+\frac{3 \theta}{2} \leqslant \frac{17}{8}
$$

This result is to be compared with the recent experimental finding $\tau_{I} \sim m_{i}$ $\mid 17-18 /$.

It is finally noted that $\left(D_{I}\right)_{d}$ is independent from the ratio $\mathrm{z} / \mathrm{m}_{I}$ [Eq. (14b)] in agreement with the observations concerning the confinement time $\tau_{I} / 18 /$. 


\section{CONCLUSIONS}

We have demonstrated that nonlocal Compton and induced scattering of drift waves /1-3/ play an essential role in the interpretation of anomalous impurity transport. In particular the numerical results derived on the basis of the theory show that

- the diffusion of high $\mathrm{z}$ impurities $\left(\mathrm{m}_{\mathrm{I}} / \mathrm{m}_{\mathbf{i}} \ll \mathrm{z}^{2}\right)$ is comparable to the base ion diffusion, and may exceed the neoclassical value as much as twe1ve times /17-19/;

- the anomalous impurity fluxes increase dramatically during the sawtooth relaxations /28-30/; these have thus a cleaning action and prevent accumulation in the core /31/;

- the impurity confinement time $\tau_{I}$ is independent from the ratio $\mathrm{Z} / \mathrm{m}_{I}$ $/ 18 /$ and increases with base ion atomic mass: $\tau_{\mathrm{I}} \sim \mathrm{m}_{\mathbf{i}}^{\mathrm{s}}, 5 / 8<\mathrm{s}<17 / 8 / 17-19 /$. A related result concerns the electron confinement time. We find that $\tau_{e}=\tau_{i} \sim m_{i}^{5 / 8} / 21 /$. 


\section{APPENDIX A}

Remarks on the dielectric function $\varepsilon\left(\vec{k}^{\prime \prime}, \omega^{\prime \prime} \rightarrow 0\right)$.

A comment is necessary concerning the expression appearing in the text. The cut-off distance of the radial eigenfunction is defined by the equation $\omega / \sqrt{2} \mathrm{k} / \|^{c}{ }_{i}=1.5 / 10 /$. Hence, in the case of nonlocal scattering,

$$
\omega_{i, \vec{k}^{\prime \prime}}^{*} / \sqrt{2}\left|k_{\mid}^{\prime \prime}\right| c_{i} \sim \omega_{i, \vec{k}}^{*} / \sqrt{2}\left|k_{\mid}\right| \mid c_{i} \sim \tau\left(1+k_{\theta^{2}}^{2}{ }^{2}\right)
$$

is typically small in terms of the temperature ratio $\mathrm{T}_{i} / \mathrm{T}_{\mathrm{e}}$ expansion, but diverges when $k_{\|}$| approaches zero. This "correction" term in the expression of $\varepsilon\left(\vec{k}^{\prime \prime}, \omega^{\prime \prime} \rightarrow 0\right)$ is to be retained whenever necessary to preserve the integrability. To make this point more precise, consider

$$
N \equiv \vec{k}^{\prime \prime} \cdot\left(k||^{\vec{k}}{ }^{\prime}+\left.k_{\eta}\right|^{\vec{k}} \perp\right) a_{i}^{2}=\tau\left[k_{\theta}^{\prime \prime} \frac{x+x^{\prime}}{L_{s}}+2 \frac{\Omega_{j}^{2}}{\omega^{2}} \frac{x x^{\prime}}{L_{s}^{2}}\left(k_{\theta} \frac{x}{L_{s}}-k_{\theta}^{\prime} \frac{x^{\prime}}{L_{s}}\right)\right]
$$

where $k_{\theta}\left(x / L_{s}\right)-k_{\theta}^{\prime}\left(x^{\prime} / L_{s}\right)=-k^{\prime \prime}$. The ratio of the first and the second term is again of the order of $\operatorname{tk}_{\theta}^{2} \mathrm{a}_{\mathrm{s}}^{2}$. The nominally subdominant term contributes mainly where $k_{\mid}^{\prime \prime} \rightarrow 0$ and $\left.\left|N / k^{\prime \prime}\right|\right|^{\varepsilon \mid} \sim 1 /\left(1+k_{\theta}^{2} a_{s}^{2}\right)$. The nominally dominant term contributes where $\left.k^{\prime \prime}\right|^{\oplus 0}$ and $\left|N / k^{\|}\right||\varepsilon| \sim 1 / k_{\theta}^{2} a_{s}^{2}\left(k_{\theta}^{2} a_{s}^{2}\right.$ is here identified as the largest of $k_{\theta^{2}}^{2} a_{s}^{2}$ and $\left\langle k_{\theta^{\prime}}^{\prime}{ }^{2} a_{s}^{2}\right.$ ). 


\section{FIGURE CAPTIONS}

1. Fluctuation spectrum of the poloidal electric field at the normalized radius $\rho=0.5$ of the moderate density TFR discharge.

2. Comparison of impurity anomalous diffusion and a) base ion anomalous diffusion; b) impurity neoclassical diffusion. The physical parameters are those of the moderate density discharge.

3. Comparison of impurity anomalous diffusion and a) base ion anomalous diffusion; b) impurity neoclassical diffusion. The physical parameters are those of the high density discharge.

4. Comparison of anomalous and neoclassical impurity friction coefficients for the physical parameters of the moderate density discharge. 


\section{REFERENCES}

1. Rogister, A., Hasselberg, G., Phys. Rev. Lett. 48 (1982) 249.

2. Rogister, A., Hasselberg, G., in Fusion Energy - 1981. (Proc. Spring Co1lege on Fusion Energy, Trieste, 1981) IAEA, Vienna (1982) 51.

3. Rogister, A., Hasselberg, G., to appear in Phys. Fluids.

4. Mazzucato, E., Phys. REv. Lett. 36 (1976) 792; Goldston R.J., Mazzucato, E., Slusher, S., Surko, C.M., in P1asma Physics and Controlled Nuclear Fusion Research (Proc. 6th Int. Conf. Berchtesgaden, 1976) Vol. I, IAEA, Vienna (1977) 371.

5. TFR Group, in Controlled Fusion and P1asma Physics (Proc. 8th Eur. Conf. Prague, 1977) Vo1. I, Institute of P1asma Physics, Prague (1978)2.

6. Mazzucato, E., Phys. Fluids 21 (1978) 1063.

7. Mazzucato, E., Phys. Rev. Lett., 48 (1982) 1828.

8. Semet, A., Mase, A., Peebles, W.A., Luhmann, N.C., Zweben, S., Phys. Rev. Lett. 45 (1980) 445.

9. Rogister, A., Hasselberg, G., Waelbroeck, F., submitted to Phys. Rev. Lett. (1983).

10. Hasselberg, G., Rogister, A., submitted to Nuc1. Fus. (1983).

11. Equipe TFR, in Plasma Physics and Controlled Nuclear Fusion Research (Proc. 8th Int. Conf. Brussels, 1980), Vol. I, IAEA, Vienna (1981) 425; a1so Report EUR-CEA-FC-1113 (1981) unpublished.

12. Manheimer, W.M., Antonsen, T.M., Phys. Fluids 22 (1979) 957.

13. Paul, J.W.M., Daughney, C.C., Holmes, L.S., Rumsby, P.T., Craig, A.D., Murray, E.L., Summers, D.D.R., Beaulieu, J., in Plasma Physics and Controlled Nuclear Fusion Research (Proc. 4th Int. Conf. Madison, 1971) Vo1. III, IAEA, Vienna (1971) 251,

14. Keilhacker, M., Kornherr, M., Niedermeyer, H., Steuer, K.H., Chodura, R., ibid. 265. 
15. Bogen, P., Dietz, K.J., Dippe1, K.H., Hintz, E., Höthker, K., Siemsen, F., Zeyer, G., ibid, 277.

16. Murakami, M., Callen, J.D., Berry, L.A., Nuc1. Fus. 16 (1976) 347. Equipe T.F.R., Nucl. Fus. 20 (1980) 1227.

17. Marmar, E.S., Rice, J.E., Allen, S.L., Phys. Rev. Lett. 45 (2025) 1980.

18. Marmar, E.S., Rice, J.E., Terry, J.L., Seguin, F.H., MIT Report PFC/JA-82-12 (1982) unpublished.

19. TFR Group, Phys. Lett. 87A. (1982) 169.

20. Engelhardt, W., Klüber, O., Meise1, D., Murmann, H., Sesnic, S., Fussman, G., Glock, E., Gottardi, N., Karger, F., Lisitano, G., Mayer, H.M., Wagner, F., in Plasma Physics and Controlled Nuclear Fusion Research (Proc. 7th Int. Conf. Innsbruck, 1979) Vol. I, IAEA, Vienna (1979) 123.

21. Hugill, J., Sheffield, J., Nucl. Fus. 18 (1978) 15.

22. Taylor, J.B., Phys. Fluids $\underline{4}$ (1961) 1142.

23. Rutherford, P.H., Phys. Fluids 17 (1974) 1782.

24. Kruskal, M.D., in Mathematical Models in Physical Sciences, Prentice Ha11, Inc., Englewood Cliffs, N.J., (1963) 17. Frieman, E.A., J. Math. Phys. 4 (1963) 410 .

25. Kadomtsev, B. B., in Plasma Turbulence, Academic Press, London (1965) 31.

26. Rogister, A., Oberman, C., J. Plasma Physics $\underline{3}$ (1969) 119.

27. We are in the process of removing this simplification from the numerical programmes developed in Ref. /10/.

28. Petrasso, R., Seguin, F.H., Loter, N.G., Marmar, E., Rice, J., MIT Report PFC/JA-82-22 (1982) unpub1ished.

29. Seguin, F.H., Petrasso, R., Marmar, E.S., MIT Report PFC/JA-82-25 (1982) unpublished.

30. Hinnov, E., Suckewer, S., et A1., Bull. Am. Phys. Soc. 25 (1980) 902. 
31. Jahns, G., Ej ima, S., Groebner, R.J., Brooks, N.H., Fisher, R.K., Hsieh, C.L., Taylor, T.S., Wesley, J.C., Fujisawa, N., Sugawara, T., Nuc1. Fus. 22 (1982) 1049.

32. A moderate variation of $G_{1}$ is not inconsistent with keeping the growth rate unchanged in view of the numerical results of Section IV B. 


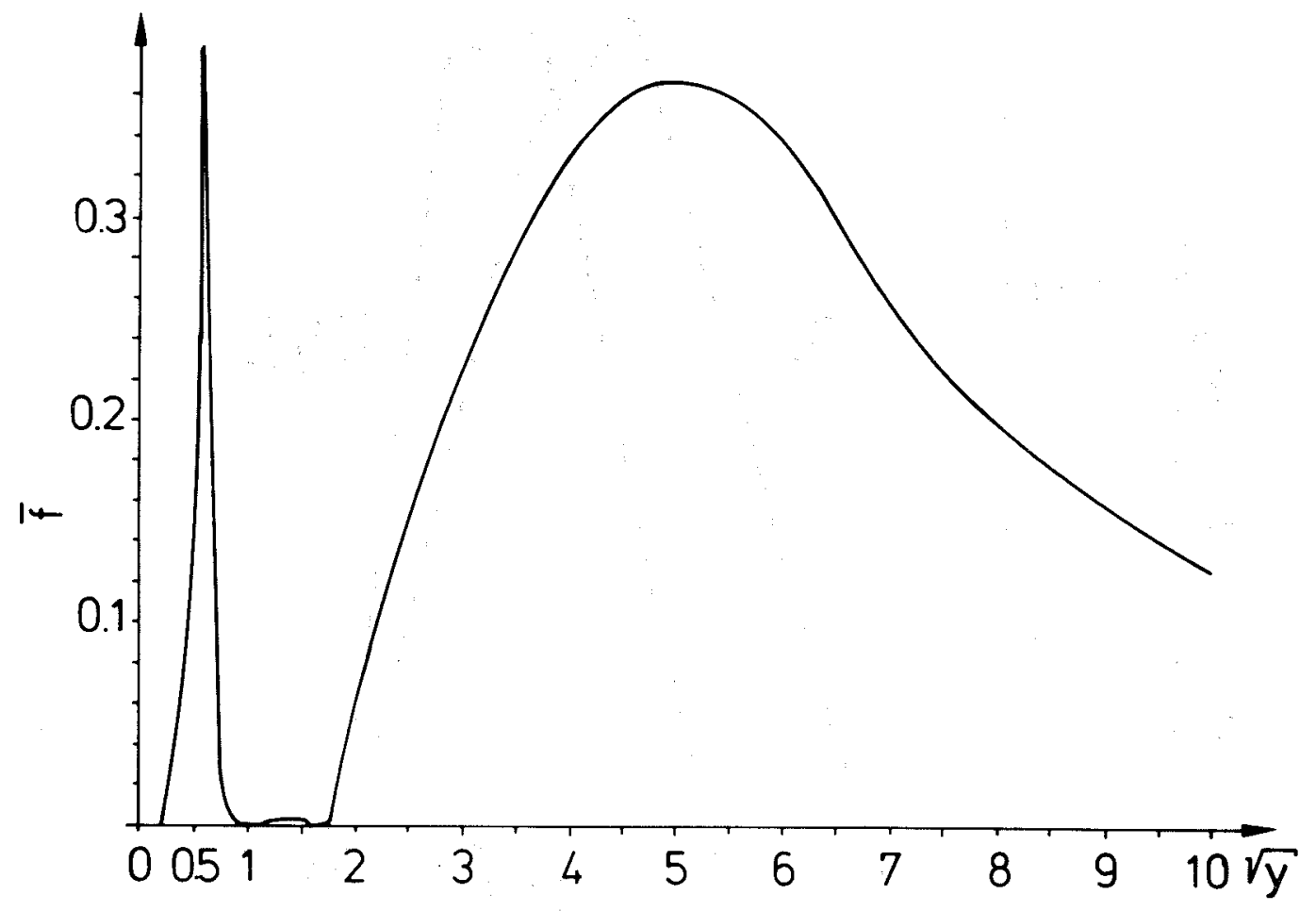

Fig. 1 


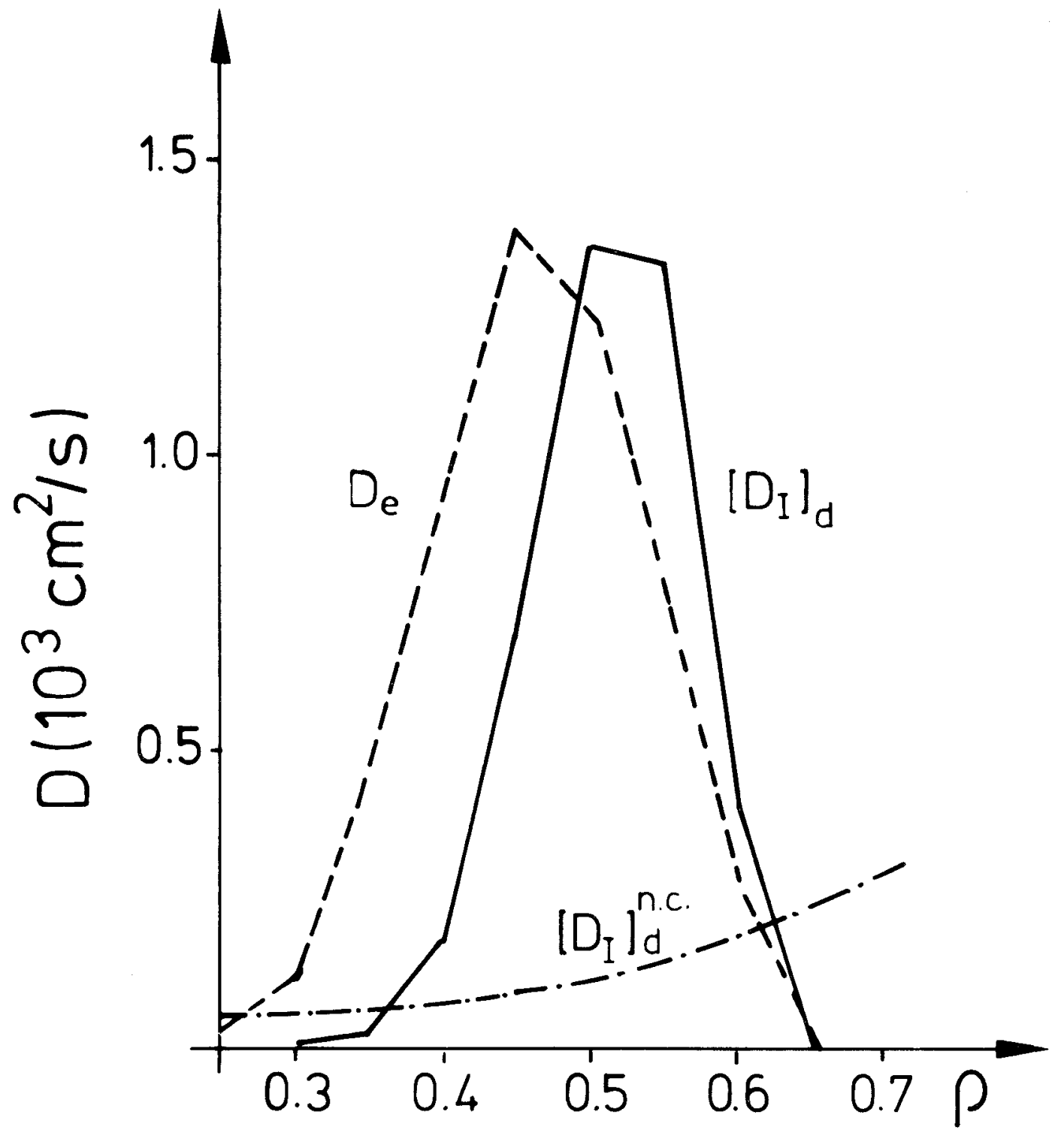

Fig. 2 


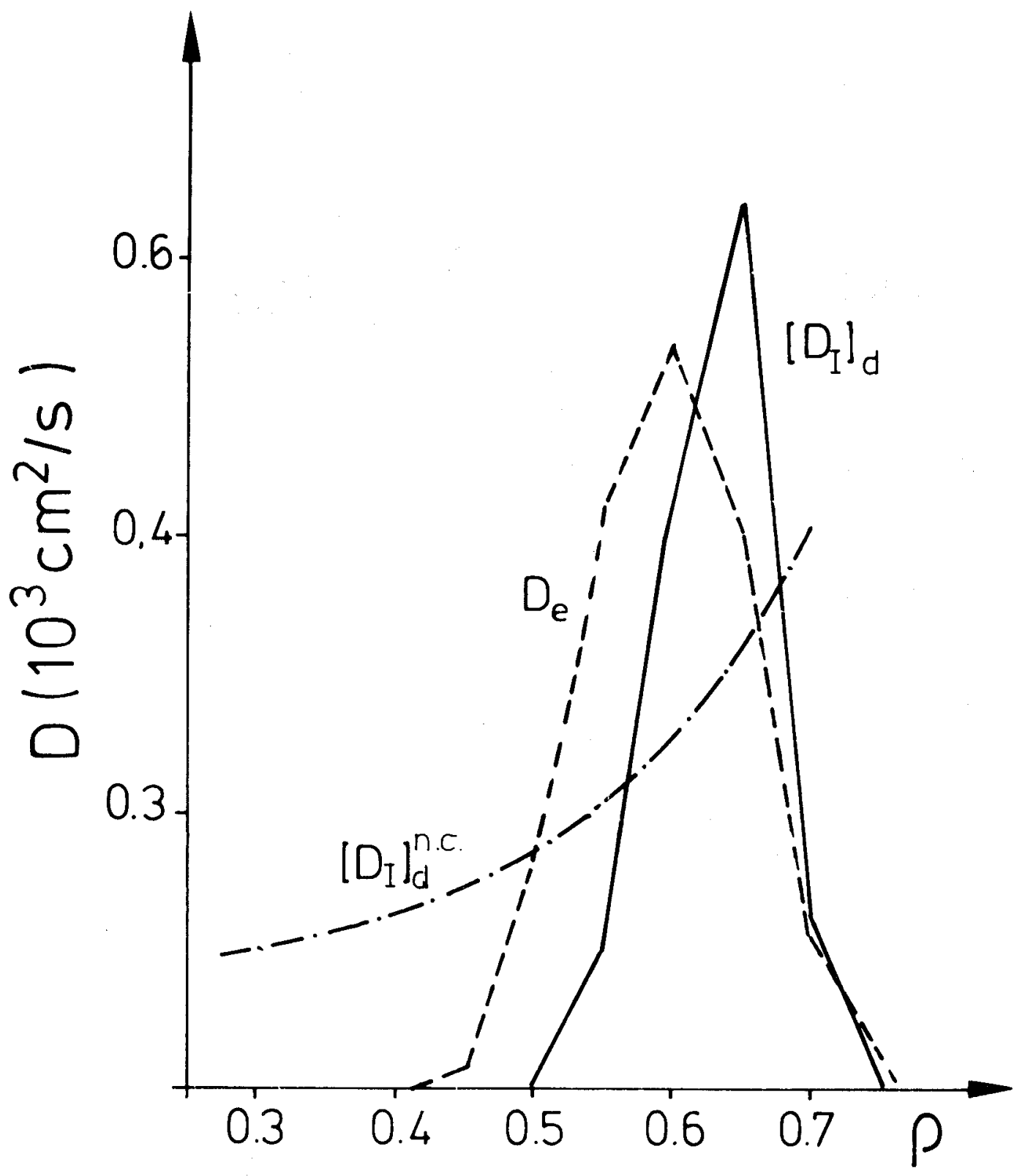

Fig. 3 


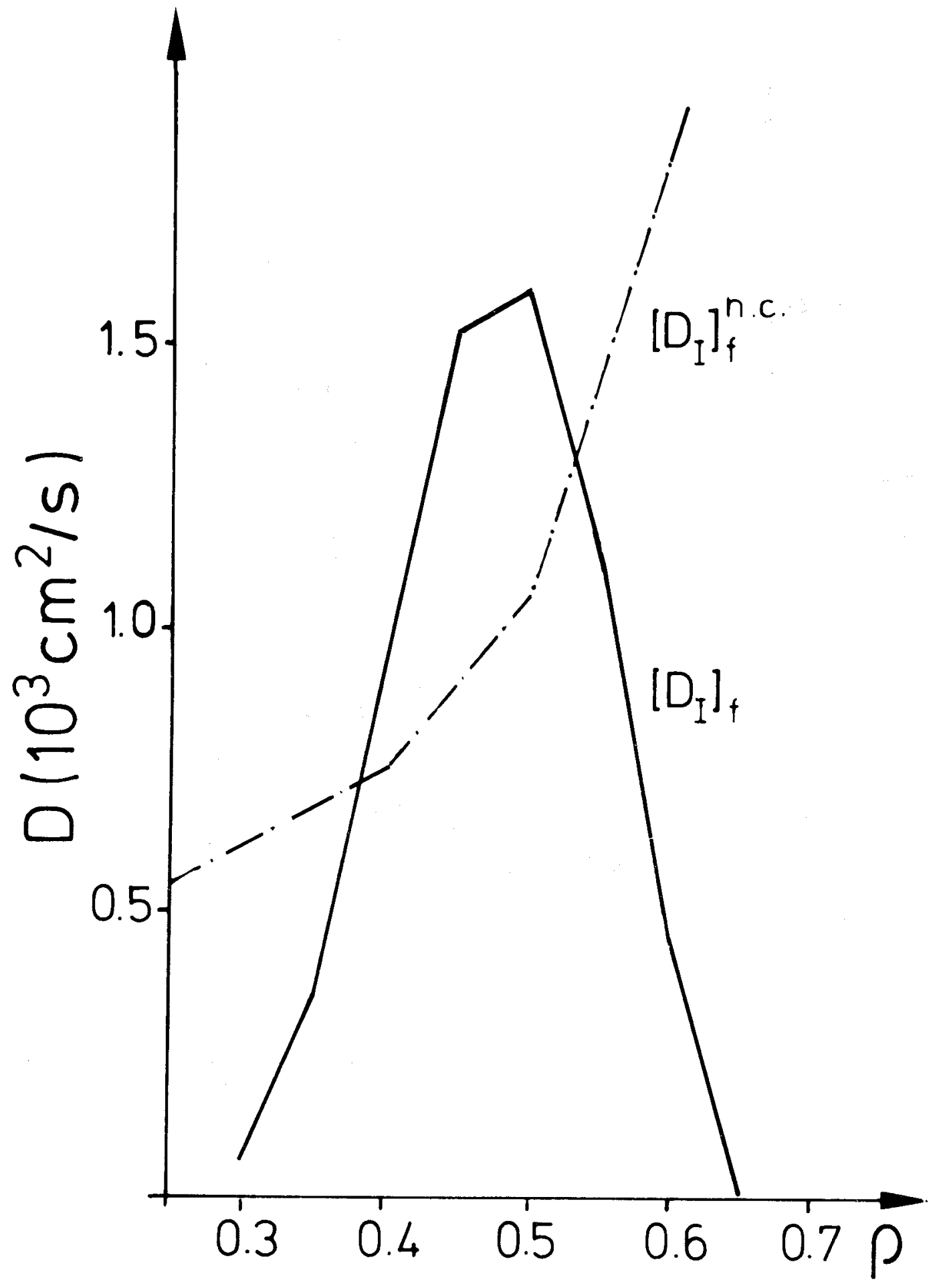

Fig. 4 